\title{
Bit and Co-ordinate Interleaved Coded Modulation
}

\author{
Md. Zafar Ali Khan and B. Sundar Rajan \\ Dept. of Electrical Comm. Engg. \\ Indian Institute of Science, Bangalore, India. \\ zafar@protocol.ece.iisc.ernet.in, bsrajan@ece.iisc.ernet.in
}

\begin{abstract}
The diversity order is crucial for the performance of communication systems over Fading Channels. It has been shown by Zehavi that the diversity and hence performance of coded modulation over fading channels can be improved by bitwise interleaving at the encoder output, and by using an appropriate soft decision metric as an input to the Viterbi decoder. A related approach is coordinate interleaving whereby the signal space coordinates are interleaved before transmission. In this paper we combine both bit interleaving and coordinate interleaving to increase the diversity order. The resulting system is referred here as $\mathrm{Bit}$ and $\mathrm{Co}-$ ordinate Interleaved Coded Modulation (BCICM). The pairwise probability of error is also derived, which shows that by proper design the diversity order can be doubled over Bit Interleaved Coded Modulation (BICM), for two dimensional signal sets. Simulation results for rate $2 / 3,4$-state 8-PSK coded modulation schemes show that BCICM significantly outperforms all known coded modulation (symbol or bit interleaved) schemes.
\end{abstract}

\section{INTRODUCTION}

The severe performance degradation over Rayleigh Fading channels is mainly determined by the deep fades of the received signal power. It is well known that multiple receiver techniques (Diversity) can be used to cope with the fading. Trellis Coded Modulation (or more generally coded Modulation) is a way of introducing time diversity [1] TCM schemes achieve significant coding gain without bandwidth expansion by treating coding and modulation as single entity. But TCM was originally designed for additive white Gaussian noise (AWGN) channels. Adaptation of TCM to fading channels resulted in design criteria for TCM over fading channels that specified that the effective length and the minimum product distance rather than Euclidean distance should be maximized for a code and the multiplicity should be minimized [7]. Several results followed this line of thought as is summarized in [7]. However despite the measures taken to improve the performance of TCM over the fading channels the improvements achieved are not so impressive as compared to TCM over AWGN channels as shown in [2].

Zehavi's [2] idea was to make the code diversity equal to the number of distinct bits(rather than channel symbols) along any error events by bitwise interleaving at the encoder output and by using an appropriate soft decision metric as the input to the Viterbi decoder. Ulf Hanson and Tor Aulin [4] generalized the concept of bit interleaving and showed that a convolution code of lower rate and hence larger hamming distance can be used giving an even higher diversity order while maintainig the spectral efficiency by expansion of channel signal constellation [4]. Al-Semari and Fuja [5] applied the concept of bit interleaving to I-Q TCM where two separate encoder are used over the two quadrature channels to almost double the code diversity. A related approach is co-ordinate interleaving whereby signal space co-ordinates of the channel symbols are interleaved before transmission [6]. This approach is less efficient over Zehavi's approach as the increase in diversity is only equal to the dimension of the channel symbols. Goeckel [8] considers the combination of BICM with rotated higher order constellations for OFDM applications.

Here we show that bit interleaving and coordinate interleaving can be combined to significantly increase the diversity order over IID Rayleigh fading channel with perfect CSI. The resulting system is referred here as Bit and coordinate interleaved coded modulation (BCICM). An upper bound on the bit error rate of 8-PSK BCICM schemes is also derived and based on this the design criteria for BCICM stems are presented. Simulation results for rate $2 / 3,4$-state 8PSK coded modulation schemes are presented to 


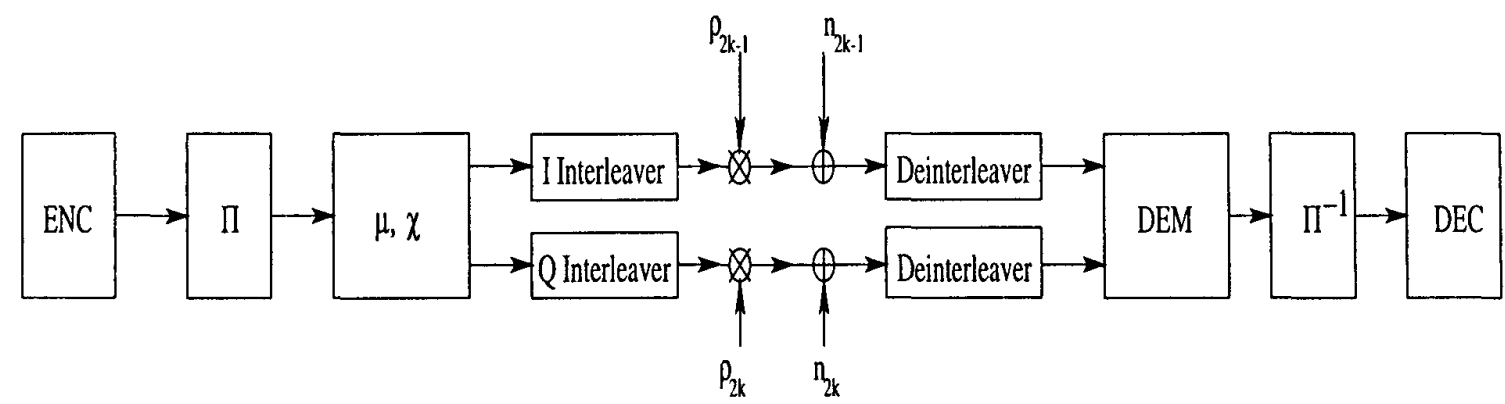

Fig. 1: System block diagram

show performance gain of BCICM over other coded modulation schemes.

\section{SYSTEM MODEL}

In this section we introduce the model of BCICM and derive its pairwise bit error probability for 8PSK signal set. The derivation follows on the line of Zehavi and familiarity with [2] will be useful.

A block diagram of proposed system is shown in Fig. 1. In general BCICM, code can be obtained from an equivalent BICM code by introducing two separate interleavers in the quadrature channels as shown in Fig. 1. Specifically the output of encoder ENC for a binary code C, is concatenated with an $\mathrm{N}$-dimensional signal set $\chi$ of size $M=2^{m}$, through a bit -interleaver $\Pi$ and a one to one binary labelling $\operatorname{map} \mu:\{0,1\}^{m} \rightarrow \chi$. The code sequence $\underline{c}$ is first interleaved by $\Pi$. Next the interleaved sequence $\Pi(c)$ is broken into sequences of $m$-bits each of which is mapped onto signals in $\chi$. Let $X_{k}=x_{2 k-1}+x_{2 k}$ be the symbol at time $\mathrm{k}$, where $x_{2 k-1}, x_{2 k}$ denote the corresponding inphase(I) and quadrature(Q) components. The resulting $\mathrm{N}$-sequence of 2-D symbols $\underline{x}=\left(X_{1}, X_{2}, \ldots, X_{N}\right)=\left\{\mathbf{x}_{\mathbf{i}}\right\}_{i=1}^{2 N}$ is passed through separate interleavers so that the $I$ and $Q$ components of the same symbol fade separately. The channel output is passed through $I$ and $Q$ de-interleavers and then through the demodulator DEM. Let $\underline{\mathbf{y}}=\left(\mathbf{Y}_{1}, \mathbf{Y}_{2}, \ldots, \mathbf{Y}_{\mathbf{N}}\right)=\left\{\mathbf{y}_{\mathbf{i}}\right\}_{\mathbf{i}=\mathbf{1}}^{\mathbf{2 N}}$ be the output of the co-ordinate interleavers corresponding to the sequence $\mathrm{x}$. This yields,

$$
Y_{k}=\rho_{k} \cdot X_{k}+n_{k}
$$

where $\rho_{k}$ is a $2-\mathrm{D}$ vector whose components are mutually independent random variables representing the amplitude of the received fading signal, $n_{k}$ is additive white Gaussian noise and - represents point wise multiplication.

Let $\chi_{b}^{i}$ be the subset of $\chi$ consisting of elements $\mathrm{X}$ whose labels have the value $b \in\{0,1\}$ in the position $\mathrm{i}$ and let $x_{I}, x_{Q}$ be the inphase and quadrature components of the symbol $\mathrm{X}=\left(x_{I}, x_{Q}\right)$ similarly $Y_{k}=\left(y_{2 k}, y_{2 k-1}\right)$ and $\rho_{k}=\left(\rho_{2 k}, \rho_{2 k-1}\right)$. The demodulator DEM produces the set of bit metrics

$$
\lambda^{i}\left(Y_{k}, \chi_{b}^{i}, b\right)=-\max _{X \in \chi_{b}^{i}}\left\|Y_{k}-\rho_{\mathbf{k}} X\right\|^{2}
$$

for $b \in\{0,1\}$ and $\mathrm{i}=1, \ldots, \mathrm{m}$. The bit metrics are passed through the metric de-interleaver and finally through the ML decoder DEC which makes decisions according to the rule

$$
\begin{aligned}
& \hat{\hat{c}}=\arg \max _{\underline{c} \in C} \sum_{i, k} \lambda^{i}\left(Y_{k}, \chi_{c}^{i}, c_{k}^{i}\right) \\
& =\min _{\underline{c} \in C} \sum_{k=1}^{N} \sum_{i=1}^{m} \underset{X \in \chi_{c}^{i}}{\max }\left\|Y_{k}-\rho_{\mathbf{k}} X\right\|^{2} \\
& =\min _{\underline{c} \in C} \sum_{k=1}^{2 N} \sum_{i=1}^{m} \max _{X \in \chi_{c}^{i}}\left\|y_{k}-\rho_{\mathbf{k}} x\right\|^{2} \\
& =M(y, \underline{c} ; \rho)
\end{aligned}
$$

where $x$ is $x_{i n}$ or $x_{q}$.

\section{Chernoff Bound}

To find an upper bound on the error performance we compute the pairwise error probability $P(\underline{c} \rightarrow$ $\underline{\hat{c}} / \underline{c}, \underline{\rho}$ ), which represents the probability of choosing the coded sequence $\underline{\hat{c}}=\left(\hat{c}_{0}, \hat{c}_{1}, \ldots, \hat{c}_{N}\right)$ instead of $\underline{\mathrm{c}}$, given the fade sequence $\rho$ (perfect CSI available, phase compensated), using the Chernoff bound technique. Thus,

$$
P(\underline{c} \rightarrow \underline{\hat{c}} / \underline{c}, \underline{\rho}) \leq E_{\mathbf{x} / \underline{c}, \rho} E_{\mathbf{y} / \mathbf{x}, \underline{\underline{c}, \underline{\rho}}}
$$




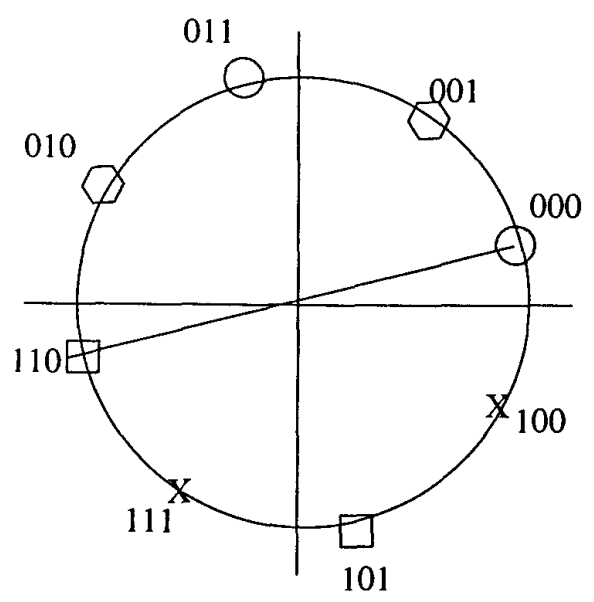

Fig. 2: Rotated 8-PSK signal set with gray labelling used in this paper

$$
e^{\lambda[M(\mathbf{y}, \underline{\hat{c}} ; \underline{\rho})-M(\mathbf{y}, \underline{c} ; \underline{\rho})]}
$$

The right hand-side of the above equation can be written as

$$
\min _{\lambda \geq 0} \prod_{k=1}^{N} \prod_{i=1}^{m} E_{X_{k}^{i} / c_{k}^{i}, \rho_{k}^{i}} E_{Y_{k}^{i} / X_{k}^{i}, \rho_{k}} e^{\lambda \Delta \lambda^{i}\left(Y_{k}^{i}, c_{k}^{i}, c_{k}^{i} ; \rho_{k}\right)}
$$

where $\Delta \lambda^{i}\left(Y_{k}^{i}, \hat{c_{k}^{i}}, c_{k}^{i} ; \rho_{k}^{i}\right)=\lambda^{i}\left(Y_{k}^{i}, \chi_{a}^{i}, a\right)-$ $\lambda^{i}\left(Y_{k}^{i}, \chi_{b}^{i}, b\right)$. Let $\hat{X} \in \chi_{c}^{i}$ and $X \in \chi_{0}^{i}$ be the solutions for (2). After substituting $a=c, b=0$ in (3), we have

$$
\begin{gathered}
\Delta \lambda^{i}\left(Y_{k}^{i}, c, 0 ; \rho_{k}^{i}\right)=-\rho_{2 k}^{2}\left(x_{i n}^{\hat{2}}-x_{i n}^{2}\right) \\
-\rho_{2 k-1}^{2}\left(\hat{x_{q}^{2}}-x_{q}^{2}\right)+2 \rho_{2 k}^{2}\left(y_{2 k}^{i}\left(x_{i n}-x_{i n}\right)\right. \\
+2 \rho_{2 k-1}^{2}\left(y_{2 k-1}^{i}\left(\hat{x_{q}}-x_{q}\right)\right.
\end{gathered}
$$

The results till now are general and applicable to all signal sets. We now evaluate the above expression for 8-PSK signal set with gray labelling shown in Fig. 2. Following an approach similar to that given Zehavi [2] it can be shown that

$$
\begin{array}{r}
E_{X_{k}^{1} / c^{1}=0} E_{Y_{k}^{1} / X_{k}^{1}, \rho_{k}^{1}} e^{\lambda \Delta \lambda^{1}\left(Y_{k}^{1}, 1,0 ; \rho_{k}^{1}\right)} \leq \\
\sum_{j=1}^{2} e^{-\frac{\rho_{2}^{2}\left\|x_{i n}(j)-x_{i n}(0)\right\|^{2}}{8 \sigma^{2}}} e^{-\frac{\rho_{1}^{2}\left\|x_{q}(j)-x_{q}(0)\right\|^{2}}{8 \sigma^{2}}}
\end{array}
$$

where

$$
x(l)=\sqrt{E_{s}} \exp \left(j \frac{l \pi}{4}\right), l=1, \ldots, 7 .
$$

Similarly, we have

$$
\begin{gathered}
E_{X_{k}^{2} / c^{2}=0, \rho} E_{Y_{k}^{2} / X_{k}^{2}, \rho} e^{\lambda \Delta \lambda^{1}\left(Y_{k}^{1}, 1,0 ; \rho_{k}^{1}\right)} \leq \\
\sum_{j=1}^{2} \frac{2 j-1}{2} e^{-\frac{\rho_{4}^{2}\left\|x_{i n}(j)-x_{i n}(0)\right\|^{2}}{8 \sigma^{2}}} e^{-\frac{\rho 2^{2}\left\|x_{q}(j)-x_{g}(0)\right\|^{2}}{8 \sigma^{2}}}
\end{gathered}
$$

The expression for $i=3$, is same as that of the above equation. Substituting these equations back in (5), we have

$$
\begin{gathered}
P(\underline{c} \rightarrow \underline{\hat{c}} / \underline{c}) \leq E_{\underline{\rho}} \prod_{k=1}^{\infty} D_{1}\left(\rho_{k}^{1}\right) D_{2}\left(\rho_{k}^{2}\right) D_{2}\left(\rho_{k}^{3}\right) D_{3}\left(\rho_{k}^{4}\right) \\
D_{4}\left(\rho_{k}^{5}\right) D_{4}\left(\rho_{k}^{6}\right)
\end{gathered}
$$

where

$$
\begin{gathered}
D_{1}(a)=\left(1 \oplus c_{k}^{i} \oplus \hat{c_{k}^{i}}\right)+\left(c_{k}^{i} \oplus \hat{c_{k}^{i}}\right) \\
\cdot\left\{D^{\left.a^{2} E_{s}\left(1-\cos \left(\frac{\pi}{4}\right)\right)^{2}+D^{a^{2} E_{s}}\right\}}\right. \\
D_{2}(a)=\left(1 \oplus c_{k}^{i} \oplus \hat{c_{k}^{i}}\right)+\frac{1}{2}\left(c_{k}^{i} \oplus \hat{c_{k}^{i}}\right) \\
\cdot\left\{D^{a^{2} E_{s}\left(1-\cos \left(\frac{\pi}{4}\right)\right)^{2}}+3 D^{a^{2} E_{s}}\right\} \\
D_{3}(a)=\left(1 \oplus c_{k}^{i} \oplus \hat{c_{k}^{i}}\right)+\left(c_{k}^{i} \oplus \hat{c_{k}^{i}}\right) \\
\cdot\left\{D^{a^{2} E_{s} \sin \left(\frac{\pi}{4}\right)^{2}}+D^{a^{2} E_{s}}\right\} \\
D_{4}(a)=\left(1 \oplus c_{k}^{i} \oplus \hat{c_{k}^{i}}\right)+\frac{1}{2}\left(c_{k}^{i} \oplus \hat{c_{k}^{i}}\right) \\
\cdot\left\{D^{a^{2} E_{s} \sin \left(\frac{\pi}{4}\right)^{2}}+3 D^{a^{2} E_{s}}\right\}
\end{gathered}
$$

where $\oplus$ is modulo 2 operation, $D=\exp \left(-1 / 8 \sigma^{2}\right)$, and $\rho_{k}^{i}$ are the fade amplitudes corresponding to the coded bit $c_{k}^{i}$. Averaging the above equation with respect to phase, $\rho_{k}^{i}$ (Rayleigh distributed) we obtain,

$$
\begin{aligned}
P(\underline{c} \rightarrow & \underline{\hat{c}} / \underline{c}) \leq\left[E_{\rho_{k}^{1}} D_{1}\left(\rho_{k}^{1}\right)\right]^{W_{1}}\left[E_{\rho_{k}^{2}} D_{2}\left(\rho_{k}^{2}\right)\right]^{W_{2}} \\
& {\left[E_{\rho_{k}^{3}} D_{2}\left(\rho_{k}^{2}\right)\right]^{W_{3}}\left[E_{\rho_{k}^{4}} D_{3}\left(\rho_{k}^{4}\right)\right]^{W_{4}} } \\
& {\left[E_{\rho_{k}^{5}} D_{4}\left(\rho_{k}^{5}\right)\right]^{W_{5}}\left[E_{\rho_{k}^{6}} D_{4}\left(\rho_{k}^{6}\right)\right]^{W_{6}} } \\
\leq & \bar{D}_{1}{ }^{W_{1}}{\overline{D_{2}}}^{W_{2}+W_{3}}{\overline{D_{3}}}^{W_{4}}{\overline{D_{4}}}^{W_{5}+W_{6}}
\end{aligned}
$$

where

$$
\bar{D}_{1}=\frac{1}{1+\frac{\bar{E}_{2}}{N_{0}} \sin ^{4}\left(\frac{\pi}{8}\right)}+\frac{1}{1+\frac{\bar{E}_{0}}{4 N_{0}}}
$$




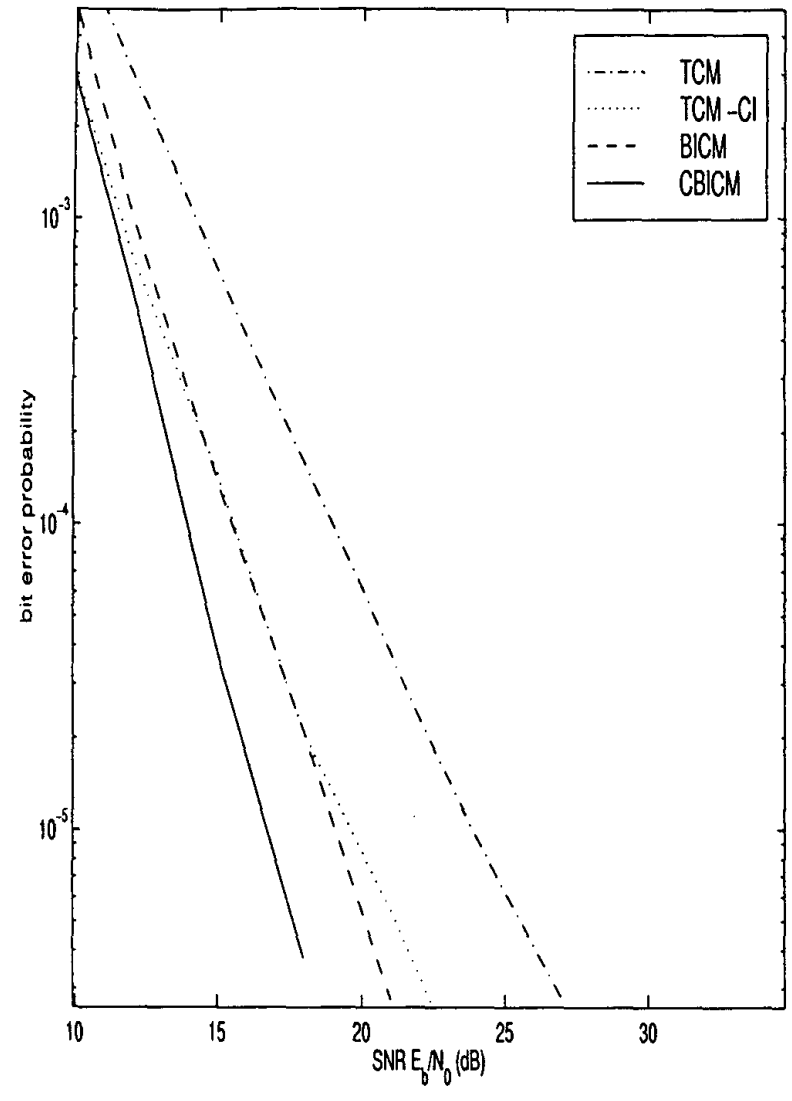

Fig. 3: Bit error rate performance of the code of this paper over a Rayleigh fading channel with perfect CSI

$$
\begin{aligned}
& \bar{D}_{2}=\frac{1}{1+\frac{\bar{E}_{0}}{N_{0}} \sin ^{4}\left(\frac{\pi}{8}\right)}+\frac{1.5}{1+\frac{\bar{E}_{s}}{4 N_{0}}} \\
& \bar{D}_{3}=\frac{1}{1+\frac{\bar{E}_{s}}{N_{0}} \sin ^{2}\left(\frac{\pi}{4}\right)}+\frac{1}{1+\frac{\bar{E}_{s}}{4 N_{0}}} \\
& \bar{D}_{4}=\frac{1}{1+\frac{\bar{E}_{0}}{N_{0}} \sin ^{2}\left(\frac{\pi}{4}\right)}+\frac{1.5}{1+\frac{\bar{E}_{s}}{4 N_{0}}}
\end{aligned}
$$

and where $\bar{E}_{s}$ is the average symbol energy, $\frac{\bar{E}_{b}}{N_{0}}=$ $\frac{\bar{E}_{s}}{R N_{0}}$ and $\mathrm{R}$ is the code rate. $W_{i}$ and $W_{i+3}$ are less than or equal to the binary hamming weight of the error path of the ith encoder output bit sequence. The equality occurs when the error path symbol co-ordinates are distinct. This can be done by rotating the signal set by an angle $\phi$ so that no two signal points have the same co-ordinate values. The optimum value of $\phi$ for 8-PSK is easily found to be $11.25^{\circ}$. For such a rotated signal set it is easily seen that $\bar{D}_{1} \geq \bar{D}_{i}, \mathrm{i}=2,3,4$. Substituting this in
(9) we have the simplified expression for pairwise error probability

$$
P(\underline{c} \rightarrow \underline{\hat{c}} / \underline{c}) \leq{\overline{D_{1}}}^{2 d_{h}}
$$

where $d_{h}$ is the hamming distance of the code. An upper bound on the probability of bit error can be found using the generating function $T\left(W_{1}, W_{2}, W_{3}, I\right)$ defined by Zehavi [2] as

$$
P_{b} \leq\left.\frac{1}{4} \frac{\partial T\left(W_{1}, W_{2}, W_{3}, I\right)}{\partial I}\right|_{W_{i}=\bar{D}_{i}, I=1} .
$$

The cutoff rate, $\tilde{R}_{0}$ can now be written as,

$\tilde{R}_{0}=3-\log _{2}\left(1+\bar{D}_{1}+\bar{D}_{3}\right)-2 \log _{2}\left(1+\bar{D}_{2}+\bar{D}_{4}\right)$.

\section{Code Design and Simulation RESULTS}

It is clear from the above equations that as with the co-ordinate interleaved TCM maximum diversity is achieved when the signal constellation points differ in all the co-ordinates. This can be achieved by rotation of the signal set. As a result of this rotation individual $\hat{D}_{i}$ 's decrease but the hamming weights are maximized. Therefore, we infer that the design criteria for BCICM systems combine the design criteria for BICM systems (as given in [3]) and co-ordinate interleaved TCM [6].

Fig. 3, shows the simulation results for rate $2 / 3$ 4-state 8-PSK coded schemes over Rayleigh fading channel with perfect CSI. The bit error performance of best 4-state TCM is shown by the label "TCM" [7]. Considerable SNR improvement is achieved by using interleaving as is seen from the graph for both bit interleaved coded modulation, "BICM", and TCM with co-ordinate interleaving, "TCM-CI". The 8-PSK signal set for "TCM-Cl" was rotated by an angle $\phi=11.25^{\circ}$ as in [6]. Also note that for 4-state 8-PSK, BICM and TCM with co-ordinate interleaving have comparable performance. The bit error performance of bit and co-ordinate interleaved coded modulation, "CBICM", with the 8-PSK signal set rotated by an angle $\phi=11.25^{\circ}$ is also plotted. It is clearly seen that as a result of the increased diversity the BICM 4-state code gives a clear 3-dB gain over the equivalent BICM scheme.

It is also worth mentioning that 8-PSK 4-state Bit-Interleaved I-Q TCM, achieves similar performance but requires two encoders and decoders. BCICM doubles the diversity, for two dimensional 
signal sets, at the cost of a little delay as a result of interleaving. Another disadvantage of this scheme is that the transmitted symbol no longer has the constant envelope property due to co-ordinate interleaving.

\section{REFERENCES}

[1] D. Divsalar and M. K. Simon, "The design of trellis coded MPSK for fading channels: Performance criteria," IEEE Trans. on Commun., vol. COM-36, No. 9, pp. 1004-1012, Sept. 1988.

[2] E. Zehavi, " 8-PSK trellis codes for a Raleigh channel," IEEE Trans. Commun., vol. 40, pp. 873-884, May 1992.

[3] G. Caire, G. Taricco and E. Biglieri, "BitInterleaved Coded Modulation," IEEE Trans. on Information theory, vol. 44, No. 3,May 1998.
[4] U. Hansson and T. Aulin, "Channel symbol expansion diversity-Improved coded modulation for the Rayleigh Fading channel," Int. Conference on communications, ICC'96., Dallas TX,June 23-27, 1996.

[5] S. A. Al-Semari and T. Fuja, "Bit interleaved I-Q TCM," ISITA'96.,Victoria, B.C., Sept 1720, 1996.

[6] B.D.Jelicic and Sumit Roy," "Design of Trellis coded QAM for Flat Fading and AWGN channel," IEEE Trans. on Veh. Technology, Vol. 44, pp.192-201, Feb. 1995.

[7] S. H. Jamali and T. LeNgoc, "Codedmodulation techniques for fading channels," Kluwer Academic Publishers, 1994.

[8] D. Goeckel, "Coded modulation with nonstandard signal sets for wireless OFDM systems," Proc. of ICC'99, pp. 791-795. 